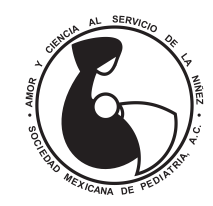

Vol. 87, No. 2 - Marzo-Abril 2020. pp 58-64 https://dx.doi.org/10.35366/94169

Revista Mexicana de

\title{
Terapia de soporte en bronquiolitis aguda grave en una Unidad de Cuidado Intensivo Pediátrico
}

\author{
Pablo Vásquez-Hoyos, ${ }^{\ddagger}, *$ Javier Mauricio González-Rincón, ${ }^{\ddagger}$ \\ Andrés Felipe Gallego-Salazar, ${ }^{\ddagger}$ Elizabeth Sánchez-Heredia, ${ }^{\ddagger}$ \\ German Arturo Latorre-Montoya ${ }^{\ddagger}$ \\ ‡ Departamento de Pediatría. Fundación Universitaria de Ciencias de la Salud. Hospital de San José. Bogotá, Colombia.
}

\begin{abstract}
RESUMEN
Objetivo: Describir el manejo de pacientes con bronquiolitis en una Unidad de Cuidado Intensivo Pediátrico (UCIP), donde se utilizan las recomendaciones de las guías clínicas basada en evidencia (GPC), en las cuales la terapia de soporte es el tratamiento básico. Material y métodos: Estudio descriptivo de una muestra consecutiva de niños que ingresaron por bronquiolitis aguda y que fueron atendidos en una UCIP entre junio de 2016 y diciembre de 2017. Se recolectó la información demográfica, la condición clínica, factores de riesgo, el manejo médico y la evolución hasta el egreso de los pacientes. Resultados: Se identificaron 150 pacientes que cumplieron los criterios de selección. La mediana de edad fue de cuatro meses (RIQ 2 - 9 meses); el $63.6 \%$ fueron hombres. Se observó una reducción del uso de medicamentos en el manejo de la UCIP, respecto al manejo previo. El uso de ventilación mecánica invasiva ocurrió en el 18.2\% ( $n=27)$ de los pacientes y cánulas nasales de alto flujo en $76.8 \%$. Se detectó co-infección bacteriana en el 19.3\%. Seis pacientes fallecieron (4\%). Conclusión: En pacientes con bronquiolitis grave, cuando se adhiere a las GPC se disminuye el uso de medicamentos innecesarios.
\end{abstract}

Palabras clave: Bronquiolitis aguda, guías de práctica clínica, tratamiento, pronóstico, lactantes.

\begin{abstract}
Objective: To describe the management of patients with bronchiolitis in a Pediatric Intensive Care Unit (PICU), where the recommendations of the evidence-based clinical practice guidelines (CPG) are used, in which support therapy is the basic treatment. Material and methods: Descriptive study of a consecutive sample of children who were admitted for acute bronchiolitis and treated in a PICU, between June 2016 and December 2017. Demographic information, clinical condition, risk factors, medical management and evolution were collected, until discharge of patients. Results: 150 patients who met the selection criteria were identified. The median age was four months (IQR 2 - 9 months); 63.6\% were men. A reduction in the use of medications in managing the PICU, compared to the previous management was observed. The use of invasive mechanical ventilation occurred in $18.2 \%$ $(n=27)$ and high-flow nasal cannulas in $76.8 \%$. Bacterial co-infection was detected in $19.3 \%$. Six patients died (4\%). Conclusion: In patients with severe bronchiolitis the use of unnecessary medications is reduced when adhered to the $\mathrm{CPG}$.
\end{abstract}

Keywords: Acute bronchiolitis, clinical practice guidelines, treatment, prognosis, infants.

\footnotetext{
* Correspondencia: PVH, pvasquez@fucsalud.edu.co

Conflicto de intereses: Los autores declaran que no tienen. Citar como: Vásquez-Hoyos P, González-Rincón JM, GallegoSalazar AF, Sánchez-Heredia E, Latorre-Montoya GA. Terapia de soporte en bronquiolitis aguda grave en una Unidad de Cuidado Intensivo Pediátrico. Rev Mex Pediatr. 2020; 87(2):58-64. doi: $10.35366 / 94169$

[Support therapy for severe acute bronchiolitis in a Pediatric Intensive Care Unit]
}

\section{INTRODUCCIÓN}

La bronquiolitis aguda es una infección viral del tracto respiratorio inferior que se caracteriza por la obstrucción de las vías aéreas de pequeño calibre, producto de la inflamación, edema y del desprendimiento de las células del epitelio respiratorio. ${ }^{1-5}$ La bronquiolitis representa uno de los principales motivos de consulta y hospitalización en pacientes menores de un año. ${ }^{1,2}$ 
El diagnóstico se hace mediante la historia clínica y el examen físico, ${ }^{2-6}$ sin necesidad de estudios paraclínicos. ${ }^{7}$ El cuadro clínico se caracteriza por síntomas catarrales, tos persistente y, posteriormente, la aparición de signos de dificultad respiratoria, como sibilancias o estertores a la auscultación.

Se han identificado diferentes factores de riesgo asociados a bronquiolitis grave, como displasia broncopulmonar, cardiopatía con repercusión hemodinámica, inmunodeficiencia o desórdenes neuromusculares. ${ }^{1,5,6,8}$

En cuanto al tratamiento, la evidencia apoya la premisa de "menos es más"; sólo las medidas de soporte son las únicas que han mostrado impacto sobre la enfermedad. Dichas medidas son nutrición e hidratación adecuadas, además de soporte con oxígeno o apoyo ventilatorio suplementario en pacientes que lo requieran, según su gravedad. ${ }^{3-5}$ Por fortuna, la gran mayoría mejora sin importar el tipo de intervención. ${ }^{2}$

A nivel mundial, se describen intervenciones para el manejo de bronquiolitis que no confieren alguna ventaja, como broncodilatadores, esteroides, antibióticos o adrenalina. ${ }^{9-13}$ También es frecuente el uso de imágenes diagnósticas que aportan poco; por ejemplo, en los EEUU se ha reportado el uso de radiografías de tórax hasta en el 55\%. ${ }^{1}$ El uso de terapias que no son útiles aumenta el tiempo de estancia hospitalaria. ${ }^{13-16}$ Además, se ha documentado variabilidad en el manejo de la bronquiolitis, independientemente de la gravedad, tanto ambulatoria en pacientes hospitalizados como en Unidades de Cuidado Intensivos Pediátricos (UCIP). ${ }^{17}$ Las razones de esta variabilidad son diversas; en una encuesta a médicos generales en el Reino Unido se refirió: "tratar potenciales diagnósticos diferenciales", "por una buena evidencia de la eficacia de los medicamentos", "beneficio del efecto placebo", o "para evitar dar explicaciones a los padres". ${ }^{18}$

Un aspecto que puede explicar lo anterior es que, en general, no hay guías específicas para casos de bronquiolitis grave..$^{5,6,9,15}$ Se sabe que las Guías de Práctica Clínica (GPC) pueden ayudar a reducir el uso de medicamentos, estudios de diagnóstico y el tiempo de estancia hospitalaria. ${ }^{13,16,19,20}$ Sin embargo, estas recomendaciones no han sido estudiadas para casos de bronquiolitis grave. Por lo anterior, en la UCIP del Hospital de San José, en Bogotá, Colombia se adoptaron las recomendaciones de las GPC para todos los pacientes con bronquiolitis aguda, sin importar factores de riesgo o gravedad. Este estudio describe la experiencia del manejo de los pacientes con bronquiolitis que requieren cuidados intensivos, haciendo comparación de las acciones realizadas antes de su ingreso a la UCIP.

\section{MATERIAL Y MÉTODOS}

Se realizó un estudio observacional y retrospectivo en una muestra consecutiva de niños menores de dos años con criterios de bronquiolitis aguda, que ingresaron a la UCIP del Hospital de San José durante el periodo de junio de 2016 a diciembre de 2017. Se excluyeron pacientes con historia de episodios previos de sibilancia. reportados por los padres o en la historia clínica.

El Hospital de San José es un hospital general de alta complejidad que atiende a una población asegurada contribuyente. La UCIP recibe casos del propio hospital, como de otros hospitales que no cuentan con cuidados intensivos. Los criterios de ingreso siguen las directrices propuestas por la Asociación Colombiana de Medicina Crítica y Cuidado Intensivo. ${ }^{21}$ Basado en la evidencia hasta ese momento, desde su apertura en 2012 se decidió otorgar exclusivamente medidas de soporte para pacientes con bronquiolitis, lo cual coincide con las GPC colombianas, publicadas en $2014 .{ }^{6}$

La información del estudio se recolectó a partir de los expedientes, desde el ingreso al hospital, durante la estancia en la UCIP y hasta el egreso hospitalario. Se recolectaron variables demográficas, saturación de oxígeno $\left(\mathrm{SaO}_{2}\right)$, fracción inspirada de oxígeno $\left(\mathrm{FiO}_{2}\right)$, forma de alimentación o hidratación, uso de soporte ventilatorio u oxígeno suplementario. También se registraron los factores asociados a gravedad (malformación de la vía aérea, condición, nutrición, enfermedad pulmonar crónica, enfermedad neuromuscular, enfermedad cardiaca con repercusión hemodinámica, y entre menores de seis meses historia de prematuridad).

Se documentó la gravedad clínica con la escala de asma modificada de Wood (M-WCAS), ${ }^{22,23}$ y con la escala para lactantes con infección respiratoria aguda (ReSViNET). ${ }^{24}$ Otro criterio de gravedad se basó en el índice $\mathrm{SaO}_{2} / \mathrm{FiO}_{2}$.

Además se registraron los estudios de imagen y de laboratorio solicitados; dentro de estos últimos se incluyeron pruebas virales y cultivos bacteriológicos. Por último, se consideró el manejo médico recibido.

Los desenlaces principales fueron: tiempo de estancia hospitalaria, complicaciones (por ejemplo, infección bacteriana agregada tanto pulmonar como extrapulmonar, neumotórax, estridor postextubación) y mortalidad.

Análisis estadístico. Los datos se presentan según su naturaleza y distribución; las variables cualitativas 
con frecuencias absolutas y relativas. Mientras que las variables cuantitativas, debido a que no cumplieron el supuesto de normalidad, se resumen con mediana e intervalos o rango intercuartílico (RIQ). Se realizó comparación entre el grupo de antes y durante con $\chi^{2}$ o prueba exacta de Fisher. Los diferentes análisis estadísticos se llevaron a cabo con el paquete Stata versión 13. Se consideró una $\mathrm{p}<0.05$ como estadísticamente significativa.

Esta investigación fue aprobada por el Comité de Investigaciones y el Comité de Ética en Investigación con seres humanos de la Fundación Universitaria de Ciencias de la Salud y del Hospital de San José. Se eximió el consentimiento informado por tratarse de un estudio sin riesgo, dado que la investigación fue a partir de los registros clínicos.

\section{RESULTADOS}

En los 18 meses de estudio, ingresaron a la UCIP 449 pacientes. De éstos, 150 (33.4\%) cumplieron con los criterios de inclusión; 29 fueron excluidos por episodios previos de sibilancias. En total se analizaron 121 pacientes.

Como se muestra en la Tabla 1, la mediana de edad al ingreso fue de cuatro meses (RIQ 2 - 9 meses) y el 63.6\% eran hombres. De los antecedentes personales, destaca que 25 pacientes (20.7\%) fueron prematuros, el $9.1 \%$ con displasia broncopulmonar y $8.3 \%$ con bajo peso al nacer.

A su ingreso a la UCIP, el $41.3 \%$ venía derivado de Urgencias del propio hospital, y el 13.2\% de salas de hospitalización.

La mediana de $\mathrm{SaO}_{2}$ al ingreso fue de $93 \%$ (RIQ 90 - 96), con una mediana de $\mathrm{FiO}_{2}$ de $50 \%$ (RIQ 40-100); así la mediana $\mathrm{SaO}_{2} / \mathrm{FiO}$ fue de 181 (RIQ 99 - 237).

En 85 pacientes (70.2\%) se pudo completar las escalas de gravedad. La mediana de puntaje en la escala ReSViNET fue de 5 puntos (RIQ 4-7); mientras que con la escala M-WCAS fue de 3 puntos (RIQ 2 - 3.5), con lo cual se clasificó a 65 pacientes (76.5\%) como leves, 19 (22.3\%) como moderados y uno como grave (1.2\%). Cabe señalar que los 16 pacientes (13.2\%), quienes no se evaluaron con estas escalas, se clasificaron como graves, dado que requirieron ventilación mecánica desde el inicio.

\section{Manejo antes de su ingreso a UCIP}

Como medida de soporte respiratorio, además de los 16 con ventilación mecánica, al momento del ingreso a UCIP 54 pacientes (44.6\%) traían oxígeno en cánula nasal convencional, 27 (22.3\%) en cánula nasal de alto flujo, 15 (12.4\%) con cámara cefálica y nueve (7.4\%) con sistema Venturi.
La Tabla 2 describe los medicamentos utilizados durante la hospitalización. Además, se usaron otros medicamentos, como sulfato de magnesio en 13 casos (10.7\%), aminofilina en seis casos (4.9\%), así como alfadornasa y oseltamivir en un caso, cada uno.

Se realizó hemograma en el $69.4 \%$ de los pacientes y radiografía de tórax en el $71.1 \%$. Sólo en el $20.7 \%$ no hubo solicitud de estudios. Al ingreso a la UCIP, el $66.9 \%$ de niños recibían alimentación e hidratación vía enteral exclusivamente y el 6.5\% estaban en ayuno; sólo 15 casos ingresaron a UCIP con sonda nasogástrica (12.5\%).

\section{Manejo durante su estancia en la UCIP}

Como también se observa en la Tabla 2, tras seguir las recomendaciones de la GPC se modificó el tratamiento farmacológico que habían recibido previamente. Así, hubo reducción estadísticamente significativa del uso de la mayoría de medicamentos durante la estancia en UCIP, a excepción de que aumentó la frecuencia de micronebulizaciones con solución salina al 3\%. Mientras que en el caso del corticoide inhalado, la disminución no fue estadísticamente significativa. Cabe señalar que se utilizaron otros medicamentos como sildenafil, bosentán y heliox por complicaciones y comorbilidades asociadas.

\begin{tabular}{|c|c|c|}
\hline & $\mathrm{n}$ & $\%$ \\
\hline Edad meses, mediana (Q1, Q3) & 4 & $(2,9)$ \\
\hline \multicolumn{3}{|l|}{ Sexo } \\
\hline Masculino & 77 & 63.6 \\
\hline Femenino & 44 & 36.4 \\
\hline Prematurez & 25 & 20.7 \\
\hline Displasia broncopulmonar & 11 & 9.1 \\
\hline Peso $<2$ Kg al nacer* & 10 & 8.3 \\
\hline Presencia de apneas & 5 & 4.1 \\
\hline $\begin{array}{l}\text { Malformación cardiaca congénita con } \\
\text { repercusión hemodinámica }\end{array}$ & 2 & 1.7 \\
\hline Enfermedad neuromuscular & 2 & 1.7 \\
\hline Malformaciones de las vías respiratorias & 1 & 0.8 \\
\hline Desnutrición aguda grave & 1 & 0.8 \\
\hline
\end{tabular}




\begin{tabular}{|c|c|c|c|c|c|c|}
\hline \multirow[b]{3}{*}{ Medicamento } & \multicolumn{4}{|c|}{ Estancia en UCIP } & \multirow{3}{*}{$\frac{\text { Reducción }}{\%}$} & \multirow[b]{3}{*}{$p^{*}$} \\
\hline & \multicolumn{2}{|c|}{ Antes } & \multicolumn{2}{|c|}{ Durante } & & \\
\hline & $n$ & $\%$ & $\mathrm{n}$ & $\%$ & & \\
\hline Salina hipertónico 3\% micronebulizada & 73 & 59.8 & 85 & 69.7 & -16.4 & 0.105 \\
\hline Agonistas adrenérgicos $\beta 2$ inhalado & 53 & 43.4 & 3 & 2.5 & 94.3 & 0.000 \\
\hline Antibióticos & 44 & 36.9 & 28 & 23.8 & 35.6 & 0.010 \\
\hline Corticoide sistémico & 41 & 33.6 & 8 & 6.6 & 80.5 & 0.000 \\
\hline Adrenalina micronebulizada & 38 & 31.1 & 10 & 8.2 & 73.7 & 0.000 \\
\hline Corticoide inhalado & 13 & 10.7 & 6 & 4.9 & 53.8 & 0.094 \\
\hline Bromuro ipratropio inhalado & 10 & 8.2 & 1 & 0.8 & 90.0 & 0.010 \\
\hline
\end{tabular}

En el $37 \%$ de los casos se realizó un hemograma y radiografía de tórax en el $33 \%$. El $82.5 \%$ de los pacientes recibió alimentación enteral por más del $80 \%$ de su tiempo de estancia en UCIP. Ninguno requirió alimentación parenteral.

El 90.0\% de los pacientes utilizó una cánula nasal convencional durante algún momento de su estancia, con una mediana de uso de tres días (RIQ 2 - 4); en $76.8 \%$ se usó cánula nasal de alto flujo (CNAF) con una mediana de cuatro días (RIQ 2 - 7). Además, se agregaron otros seis pacientes que requirieron ventilación mecánica invasiva, por lo que en total fue del $18.2 \%$ $(n=22)$, siendo la mediana de ventilación de seis días (RIQ 2 - 10).

Se realizó panel viral al $98.3 \%$ de los pacientes, con resultado positivo en 68 (57.1\%); de los cuales 54 fueron positivos para virus sincicial respiratorio (VSR), nueve para virus de parainfluenza, siete para virus de influenza y cinco casos para adenovirus. En seis pacientes se aisló más de un virus.

\section{Desenlaces}

Las comorbilidades infecciosas se documentaron en 23 pacientes (19.1\%) en quienes se detectó neumonía bacteriana, además de 12 pacientes con hemocultivos positivos (9.9\%), dos infecciones de vías urinarias y un caso de otitis media aguda. Otras complicaciones fueron: dos pacientes con neumotórax asociado con ventilación mecánica y siete pacientes tuvieron estridor posterior a la extubación. La mediana de días de estancia en la UCIP fue de siete días y la estancia hospitalaria total fue de 11 días. Seis pacientes fallecieron; en la Tabla 3 se describe la información individual de cada caso.

Finalmente, de la UCIP egresaron vivos 115 pacientes $(95 \%)$, de los cuales en $47 \%$ el egreso fue directamente a casa y el $53 \%$ a una sala de hospitalización. El $62.6 \%$ egresó con oxígeno domiciliario, como es habitual en Bogotá por la altura sobre el nivel del mar.

\section{DISCUSIÓN}

Este estudio describe principalmente el manejo de bronquiolitis aguda en pacientes de una UCIP de Bogotá, en la cual se han adoptado las recomendaciones de las GPC. De esta forma, al seguir la guía se pudo reducir el uso de fármacos, sin observar complicaciones.

$\mathrm{Al}$ revisar la caracterización demográfica de la población que estudiamos, se constató que es similar a otras cohortes de pacientes descritas en la UCIP con bronquiolitis aguda. ${ }^{9,14}$ Los principales factores de riesgo para enfermedad grave fueron la historia de prematuridad y displasia broncopulmonar. Un estudio reciente con más de 132 mil pacientes confirma que la menor edad cronológica y la prematuridad son factores de riesgo asociados a hospitalización por VSR. ${ }^{25}$

En contraste con otros estudios, la gravedad de los pacientes al ingreso en su mayoría tuvo puntajes 
inferiores. ${ }^{14}$ Una explicación pudiera ser porque Bogotá se encuentra a gran altura sobre el nivel del mar $(2,600 \mathrm{~m}){ }^{6}$

En cuanto al uso de medicamentos, ninguna guía recomienda el uso rutinario de corticoides, ${ }^{26,27}$ broncodilatadores, ${ }^{27}$ antibióticos, ${ }^{28}$ inhibidores de leucotrienos ${ }^{29} \mathrm{o}$ antivirales. ${ }^{30} \mathrm{La}$ adrenalina micronebulizada ha mostrado en algunos estudios superioridad al placebo en las primeras 24 horas y sólo una guía contempla su uso como terapia de rescate. ${ }^{4,31-33}$ La utilidad de la solución salina hipertónica al 3\% micronebulizada se mantiene en duda de acuerdo a meta-análisis recientes, ${ }^{34-36}$ pero algunas guías la recomiendan en pacientes hospitalizados. ${ }^{1,3,4,37}$

El presente estudio describe que, antes del ingreso a UCIP, el uso de medicamentos que se recomienda en
GPC es muy frecuente, lo cual ya ha sido documentado en España y EEUU. ${ }^{9,11,12}$ Pero también es de destacar que se usan fármacos que no se ha probado posible utilidad en bronquiolitis, tales como anticolinérgicos, sulfato de magnesio, aminofilina y alfa-dornasa.

Otra recomendación en la GPC es administrar oxígeno suplementario a los pacientes con hipoxemia persistente. Sin embargo, existen inconvenientes para seguir esta recomendación, pues se debe tomar en cuenta que los puntos de corte de pulsioximetría son arbitrarios, ya que no toman en cuenta las ciudades con gran altura, como lo es Bogotá. 6,38,39 Estudios recientes demuestran que desde que se utiliza la pulsioximetría en bronquiolitis ha aumentado de forma exponencial la tasa de hospitalización, pero sin modificar la mortalidad. ${ }^{39,40}$

\section{Tabla 3: Características de los casos de mortalidad.}

\begin{tabular}{|c|c|c|c|c|c|c|c|}
\hline Sexo y edad & Factores de riesgo & $\begin{array}{l}\text { Estancia } \\
\text { UCIP } \\
\text { (días) }\end{array}$ & $\begin{array}{c}\mathrm{O}_{2} \\
\text { ingreso }\end{array}$ & $\begin{array}{l}\text { Severidad } \\
\text { ingreso }\end{array}$ & Infeccioso & $\begin{array}{l}\text { PCR } \\
\text { máximo }\end{array}$ & Observaciones adicionales \\
\hline $\begin{array}{l}\text { Femenino } \\
6 \text { meses }\end{array}$ & $\begin{array}{l}\text { Prematuro de } 28 \\
\text { semanas, DBP se- } \\
\text { vera, DNT crónica, } \\
\text { indígena, retraso } \\
\text { global del desarrollo }\end{array}$ & 74 & VMI & $\begin{array}{l}\mathrm{SaO}_{2} / \mathrm{FiO}_{2}: \\
\quad 186\end{array}$ & $\begin{array}{l}\text { Hemocultivos ne- } \\
\text { gativos; panel viral } \\
\text { de parainfluenza } \\
\text { 3, urocultivo con E. } \\
\text { coli y Morganella }\end{array}$ & 13 & $\begin{array}{l}\text { Con hipertensión pulmonar severa } \\
\text { refractaria (sildenafil y bosentán), } \\
\text { gastrostomía y traqueostomía. } \\
\text { Estado postparo núm. } 4\end{array}$ \\
\hline $\begin{array}{l}\text { Femenino } \\
9 \text { meses }\end{array}$ & Ninguno & 4 & VMI & $\begin{array}{l}\mathrm{SaO}_{2} / \mathrm{FiO}_{2}: \\
\quad 88\end{array}$ & $\begin{array}{l}\text { Hemocultivos ne- } \\
\text { gativos, panel viral } \\
\text { VSR y adenovirus }\end{array}$ & 26 & $\begin{array}{l}\text { Ingresa con choque mixto } \\
\text { (obstructivo y cardiogénico), } \\
\text { lesión renal aguda y sospecha } \\
\text { de coinfección bacteriana }\end{array}$ \\
\hline $\begin{array}{l}\text { Masculino, } \\
18 \text { meses }\end{array}$ & $\begin{array}{l}\text { Hidrocefalia (deriva- } \\
\text { da), retraso global } \\
\text { severo del desarrollo } \\
\text { neurológico }\end{array}$ & 1 & VMI & $\begin{array}{l}\mathrm{SaO}_{2} / \mathrm{FiO}_{2}: \\
\quad 98\end{array}$ & $\begin{array}{l}\text { Hemocultivos y } \\
\text { panel viral } \\
\text { negativos }\end{array}$ & 18.5 & $\begin{array}{l}\text { Ingresa estado postparo car- } \\
\text { diaco de } 20 \text { minutos; presenta } \\
\text { hemorragia pulmonar y choque } \\
\text { refractario }\end{array}$ \\
\hline $\begin{array}{l}\text { Masculino, } 4 \\
\text { meses }\end{array}$ & $\begin{array}{l}\text { Prematuro } 24 \\
\text { semanas, DBP } \\
\text { severa }\end{array}$ & 3 & VMI & $\begin{array}{l}\mathrm{SaO}_{2} / \mathrm{FiO}_{2} \\
\quad 90\end{array}$ & $\begin{array}{l}\text { Hemocultivos } \\
\text { y panel viral } \\
\text { negativos }\end{array}$ & 2.1 & $\begin{array}{l}\text { Ingresa con falla multiorgánica, } \\
\text { incluyendo lesión renal aguda }\end{array}$ \\
\hline $\begin{array}{l}\text { Masculino, } \\
2 \text { meses }\end{array}$ & Ninguno & 7 & VMI & $\begin{array}{l}\mathrm{SaO}_{2} / \mathrm{FiO}_{2}: \\
\quad 196\end{array}$ & $\begin{array}{l}\text { Panel viral VSR, } \\
\text { hemocultivos: } K \\
\text { pneumoniae patrón } \\
\text { BLEE }\end{array}$ & 36.5 & $\begin{array}{l}\text { Coinfección bacteriana asociada } \\
\text { con cuidado de salud, traqueítis, } \\
\text { bacteriemia, y urocultivo por } \\
\text { Klebsiella pneumoniae, poste- } \\
\text { rior a fallecer }\end{array}$ \\
\hline $\begin{array}{l}\text { Masculino, } \\
\text { de } 7 \text { meses }\end{array}$ & $\begin{array}{l}\text { DNT crónica, } \\
\text { indígena }\end{array}$ & 5 & VMI & $\begin{array}{l}\mathrm{SaO}_{2} / \mathrm{FiO}_{2}: \\
\quad 174\end{array}$ & $\begin{array}{l}\text { Panel viral nega- } \\
\text { tivo, hemocultivos } \\
\text { y urocultivo por K } \\
\text { pneumoniae BLEE }\end{array}$ & 7.3 & $\begin{array}{l}\text { Ingresa con lesión renal aguda; } \\
\text { se documenta IVU y bacterie- } \\
\text { mia por Klebsiella BLEE, falla } \\
\text { multiorgánica. Deterioro antes } \\
\text { del aislamiento del germen }\end{array}$ \\
\hline
\end{tabular}


Para los pacientes que requieren un soporte ventilatorio adicional, se observó que en UCIP fue muy frecuente uso de CNAF y un bajo uso de ventilación mecánica invasiva. A nivel mundial, el uso de CNAF como medida de soporte ventilatorio no invasivo inicial ha tenido gran acogida por su efectividad y facilidad de uso. ${ }^{41,42}$ Ésta se usa habitualmente como terapia de rescate para niños con bronquiolitis aguda que no mejoran con oxígeno por cánula nasal convencional, reduciendo las tasas de intubación y los costos de la atención, además de que proporcionan al niño mayor confort y permitir mejor su alimentación. ${ }^{43-46}$ Lo cual contribuyó a que la mayoría de nuestros pacientes recibieran alimentación vía oral, lo cual se recomienda en las GPC. 3,4,47-49

Por otro lado, se pudo observar que el uso de estudios de laboratorio y de imagen fue muy frecuente. Si bien, en la mayoría de los casos con bronquiolitis no se requieren, el grupo de pacientes incluidos se consideraron con un algún dato de gravedad, por lo que estas herramientas podrían ayudar en la evaluación de cada paciente. ${ }^{2,4}$ Hasta el momento, el recuento de glóbulos blancos no ha mostrado ser útil para predecir infecciones graves en niños infectados por VRS. ${ }^{1,50}$

$\mathrm{El}$ agente etiológico más frecuentemente aislado en esta cohorte es el VSR, seguido de los virus parainfluenza e influenza, lo cual es semejante a otros estudios. ${ }^{2,51}$ La utilidad del aislamiento viral es controvertida, pues no hay un beneficio claro para determinar la etiología viral en un paciente con bronquiolitis. De hecho, en la mayoría de guías no se recomienda realizar de rutina, ya que no cambia el manejo. ${ }^{1,52,53}$ En esta unidad se realiza para comprobar el diagnóstico y evitar usar otros tratamientos.

Las infecciones bacterianas que complican la bronquiolitis deben sospecharse en pacientes con curso clínico no habitual, por ejemplo, ante el reinicio de fiebre después o cuando la evolución es tórpida. ${ }^{1} \mathrm{La}$ complicación más frecuente observada fue la co-infección bacteriana, la cual se relacionó con los desenlaces fatales observados (Tabla 3). ${ }^{53,54}$

Los principales desenlaces clínicos que se reportan en el presente estudio, son similares a los de otras cohortes en UCIP. ${ }^{9,14}$ La mortalidad estuvo relacionada con comorbilidades; ningún caso se encontró asociado de forma exclusiva con la bronquiolitis.

\section{AGRADECIMIENTOS}

A la Dra. Merideidy Plazas y al personal de salud implicado en la atención de los pacientes, enfermeras y terapeutas respiratorias, por la colaboración prestada durante la realización del estudio.

\section{REFERENCIAS}

1. Ralston SL, Lieberthal AS, Meissner HC, Alverson BK, Baley JE, Gadomski AM et al. Clinical practice guideline: the diagnosis, management, and prevention of bronchiolitis. Pediatrics. 2014; 134(5): e1474-502.

2. Florin TA, Plint AC, Zorc JJ. Viral bronchiolitis. Lancet. 2017; 389(10065): 211-224.

3. Meissner HC. Viral bronchiolitis in children. N Engl J Med. 2016; 374(1): 62-72.

4. National Institute for Clinical Excellence. Bronchiolitis: diagnosis and management of bronchiolitis in children. Clinical Guideline 9. London: NICE, 2015.

5. Friedman JN, Rieder MJ, Walton JM. Bronchiolitis: recommendations for diagnosis monitoring and management of children one to 24 months of age. Can J Pediatr. 2014; 19(9): 1485.

6. Ministerio de Salud y Protección Social. Guía de Práctica Clínica inicial de la Neumonía en niños y niñas menores de 5 años y bronquiolitis en niños y niñas menores de 2 años. Colombia, Ministerio de Salud y Protección Social; 2014.

7. Rodríguez CE, Sossa MP. Costo-efectividad de la radiografía de tórax en lactantes con sospecha clínica de bronquiolitis viral en Colombia. Rev Panam Salud Publica. 2011; 29(3): 153-161.

8. Willwerth BM, Harper MB, Greenes DS. Identifying hospitalized infants who have bronchiolitis and are at high risk for apnea. Ann Emerg Med. 2006; 48(4): 441-447.

9. Pierce HC, Mansbach JM, Fisher ES, Macias CG, Pate BM, Piedra PA et al. Variability of intensive care management for children with bronchiolitis. Hosp Pediatr. 2015; 5(4): 175-184.

10. Ochoa SC, González DJ. Manejo de la bronquiolitis aguda en atención primaria: análisis de variabilidad e idoneidad (proyecto aBREVIADo). An Pediatr. 2013; 79(3): 167-176.

11. Ochoa Sangrador C, González de Dios J. Overuse of bronchodilators and steroids in bronchiolitis of different severity. Bronchiolitis-study of variability, appropriateness, and adequacy. Allergol Immunopathol (Madr). 2014; 42(4): 307-315.

12. Ochoa Sangrador C, González de Dios J. Management of acute bronchiolitis in emergency wards in Spain: Variability and appropriateness analysis (aBREVIADo Project). Eur J Pediatr. 2012; 171(7): 1109-1119.

13. Mittal V, Darnell C, Walsh B, Mehta A, Badawy M, Morse R et al. Inpatient bronchiolitis guideline implementation and resource utilization. Pediatrics. 2014; 133(3): e730-737.

14. Essouri S, Baudin F, Chevret L, Vincent M, Emeriaud G, Jouvet P. Variability of care in infants with severe bronchiolitis: less-invasive respiratory management leads to similar outcomes. J Pediatr. 2017; 188: 156-162.

15. Carroll CL, Faustino EVS, Pinto MG, Sala KA, Canarie MF, Li S et al. A regional cohort study of the treatment of critically ill children with bronchiolitis. J Asthma. 2016; 53(10): 1006-1011.

16. McCulloh RJ, Smitherman S, Adelsky S, Congdon M, Librizzi $\mathrm{J}$, Koehn $\mathrm{K}$ et al. Hospitalist and nonhospitalist adherence to evidence-based quality metrics for bronchiolitis. Hosp Pediatr. 2012; 2 : 19-25.

17. Christakis DA, Cowan CA, Garrison MM, Molteni R, Marcuse E, Zerr DM. Variation in inpatient diagnostic testing and management of bronchiolitis. Pediatrics. 2005; 115(4): 878-884. 
18. Carande EJ, Cheung CR, Pollard AJ, Drysdale SB. Change in viral bronchiolitis management in primary care in the UK after the publication of NICE guideline. Thorax. 2018; 73: 674-676.

19. Librizzi J, McCulloh R, Koehn K, Alverson B. Appropriateness of testing for serious bacterial infection in children hospitalized with bronchiolitis. Hosp Pediatr. 2014; 4(1): 33-38.

20. Tyler A, Krack P, Bakel LA, O'Hara K, Scudamore D, Topoz I et al. Interventions to reduce over-utilized tests and treatments in bronchiolitis. Pediatrics. 2018; 141(6): e20170485.

21. Khemani RG, Thomas NJ, Venkatachalam V, Scimeme JP, Berutti $\mathrm{T}$, Schneider JB et al. Comparison of $\mathrm{SpO}_{2}$ to $\mathrm{PaO}_{2}$ based markers of lung disease severity for children with acute lung injury. Crit Care Med. 2012; 40(4): 1309-1316.

22. Rivas-Juesas C, Rius Peris J, García A, Madramany A, Peris $M$, Álvarez $L$ et al. A comparison of two clinical scores for bronchiolitis. A multicentre and prospective study conducted in hospitalised infants. Allergol Immunopathol (Madr). 2018; 46(1): 15-23.

23. Hall CB, Weinberg GA, Blumkin AK, Edwards KM, Staat MA, Schultz AF et al. Respiratory syncytial virus-associated hospitalizations among children less than 24 months of age. Pediatrics. 2013; 132(2): e341-348.

24. Destino L, Weisgerber MC, Soung P, Bakalarski D, Yan K, Rehborg $R$ et al. Validity of respiratory scores in bronchiolitis. Hosp Pediatr. 2012; 2(4): 202-209.

25. Fernandes RM, Bialy LM, Vandermeer B, Tjosvold L, Plint AC, Patel $\mathrm{H}$ et al. Glucocorticoids for acute viral bronchiolitis in infants and young children. Cochrane Database Syst Rev. 2013; (6): CD004878.

26. Gadomski AM, Scribani MB. Bronchodilators for bronchiolitis. Cochrane Database Syst Rev. 2014; (6): CD001266.

27. Farley R, Spurling GK, Eriksson L, Del Mar CB. Antibiotics for bronchiolitis in children under two years of age. Cochrane Database Syst Rev. 2014; (10): CD005189.

28. Liu F, Ouyang J, Sharma AN, Liu S, Yang B, Xiong W et al. Leukotriene inhibitors for bronchiolitis in infants and young children. Cochrane Database Syst Rev. 2015; (3): CD010636.

29. Li L, Avery R, Budev M, Mossad S, Danziger-Isakov L. Oral versus inhaled ribavirin therapy for respiratory syncytial virus infection after lung transplantation. J Hear Lung Transplant. 2012; 31(8): 839-844

30. Sakulchit T, Goldman R. Nebulized epinephrine for young children with bronchiolitis. Can Fam Physician. 2016; 62: 991-993.

31. Hartling L, Bialy LM, Vandermeer B, Tjosvold L, Johnson DW, Plint AC et al. Epinephrine for bronchiolitis. Cochrane Database Syst Rev. 2018; (6): CD003123.

32. Angoulvant F, Bellêttre X, Milcent K, Teglas JP, Claudet I, Le Guen $C G$ et al. Effect of nebulized hypertonic saline treatment in emergency departments on the hospitalization rate for acute bronchiolitis: A randomized clinical trial. JAMA Pediatr. 2017; 171(8): 1-8.

33. Brooks CG, Harrison WN, Ralston SL. Association between hypertonic saline and hospital length of stay in acute viral bronchiolitis: a reanalysis of 2 meta-analyses. JAMA Pediatr. 2016: 170(6): 577-584.

34. Maguire $\mathrm{C}$, Cantrill $\mathrm{H}$, Hind D, Bradburn M, Everard M. Hypertonic saline (HS) for acute bronchiolitis: systematic review and metaanalysis. BMC Pulm Med. 2015; 15(1): 1-17.

35. Kou M, Hwang V, Ramkellawan N. Bronchiolitis: from practice guideline to clinical practice. Emerg Med Clin North Am. 2018; 36: $275-286$.
36. Purcell K, Fergie J. Lack of usefulness of an abnormal white blood cell count for predicting a concurrent serious bacterial infection in infants and young children hospitalized with respiratory syncytial virus lower respiratory tract infection. Pediatr Infect Dis J. 2007; 26(4): 311-315.

37. Ralston SL. Could this be the last word on hypertonic saline? JAMA Pediatr. 2017; 171(8): 7-8.

38. Oakley E, Borland M, Neutze J, Acworth J, Krieser D, Dalziel S et al. Nasogastric hydration versus intravenous hydration for infants with bronchiolitis: a randomized trial. Lancet Respir Med. 2013; 1(2): 113-120.

39. Kennedy N, Flanagan N. Is nasogastric fluid therapy a safe alternative to intravenous route in infants with bronchiolitis? Arch Dis Child. 2005; 90: 320-321.

40. Ross PA, Newth CJ, Khemani RG. Accuracy of pulse oximetry in children. Pediatrics. 2014; 133(1): 22-29.

41. Quinonez RA, Coon ER, Schroeder AR, Moyer VA. When technology creates uncertainty: pulse oximetry and overdiagnosis of hypoxaemia in bronchiolitis. BMJ. 2017; 358: 1-5.

42. Vincent JO, Lo H, Wu S. Bronchiolitis care in the hospital. Rev Recent Clin Trials. 2017; 12(4): 1-8.

43. Sinha IP, McBride AK, Smith R, Fernandes RM. CPAP and highflow nasal cannula oxygen in bronchiolitis. Chest. 2015; 148(3): 810-823.

44. Franklin D, Babl FE, Schlapbach LJ, Oakley E, Craig S, Neutze $\mathrm{J}$ et al. A randomized trial of high-flow oxygen therapy in infants with bronchiolitis. N Engl J Med. 2018; 378(12): 1121-1131.

45. Kepreotes E, Whitehead B, Attia J, Oldmeadow C, Collison A, Searles A et al. High-flow warm humidified oxygen versus standard low-flow nasal cannula oxygen for moderate bronchiolitis (HFWHO RCT): an open, phase 4, randomized controlled trial. Lancet. 2017; 389 (10072): 930-939.

46. Ten Brink F, Duke T, Evans J. High-flow nasal prong oxygen therapy or nasopharyngeal continuous positive airway pressure for children with moderate to severe respiratory distress? Pediatr Crit Care Med. 2013; 14(7): e326-331.

47. Bressan S, Balzani M, Krauss B, Pettenazzo A, Zanconato S, Baraldi E. High-flow nasal cannula oxygen for bronchiolitis in a pediatric ward: a pilot study. Eur J Pediatr. 2013; 172(12): 16491656.

48. McKiernan C, Chua LC, Visintainer PF, Allen H. High flow nasal cannulae therapy in infants with bronchiolitis. J Pediatr. 2010; 156(4): 634-638

49. Mayfield S, Bogossian F, O'Malley L, Schibler A. High-flow nasal cannula oxygen therapy for infants with bronchiolitis: pilot study. J Paediatr Child Health. 2014; 50(5): 373-378.

50. Fretzayas A, Moustaki M. Etiology and clinical features of viral bronchiolitis in infancy. World J Pediatr. 2017; 13(4): 293-299.

51. Cahill AA, Cohen J. Improving evidence based bronchiolitis care. Clin Pediatr Emerg Med. 2018; 19(1): 33-39.

52. Thorburn K, Harigopal S, Reddy V, Taylor N, Van Saene HKF. High incidence of pulmonary bacterial co-infection in children with severe respiratory syncytial virus (RSV) bronchiolitis. Thorax. 2006; 61(7): 611-615.

53. Wiegers HMG, van Nijen L, van Woensel JBM, Bem RA, de Jong $\mathrm{MD}$, Calis JCJ. Bacterial co-infection of the respiratory tract in ventilated children with bronchiolitis; a retrospective cohort study. BMC Infect Dis. 2019;19(1):938.

54. Ghazaly M, Nadel S. Characteristics of children admitted to intensive care with acute bronchiolitis. Eur J Pediatr. 2018;177(6):913-20 